\title{
Idiopathic intracranial hypertension: Update on diagnosis and management
}

\author{
Authors: Benjamin R Wakerley, ${ }^{A}$ Susan P Mollan ${ }^{B}$ and Alexandra J Sinclair ${ }^{C}$
}

Idiopathic intracranial hypertension is a condition of raised intracranial pressure of unknown cause. Features include new onset headache, which is frequently non-specific; papilloedema is present, visual disturbances are common; and there may be sixth nerve palsy. Diagnosis includes brain imaging with venography to exclude structural causes and venous sinus thrombosis. Lumbar puncture reveals pressure greater than $250 \mathrm{mmCSF}$ with normal constituents. Treatments aim to modify the disease, prevent permanent visual loss and manage headaches. These include weight loss. For those with rapid visual decline, urgent surgical intervention is essential. For most, this is a chronic condition characterised by significantly disabling headaches.

\section{Introduction}

Adult idiopathic intracranial hypertension (IIH) is a rare condition of unknown cause, which results in raised intracranial pressure (ICP). ${ }^{1}$ Young obese women are most commonly affected, although rarely it may occur in men. The incidence in the UK general population is approximately 4.7 per 100,000 and this has recently increased in parallel with obesity. ${ }^{2}$ Typically patients report a migraine-like headache, visual loss or disturbance, and pulsatile tinnitus. Although the exact pathogenesis remains unknown, there is a strong association with obesity, typically centripetal, and androgen excess. ${ }^{3,4}$ IIH patients also have double the normal cardiovascular risk, independent of obesity. ${ }^{1,5}$ In most patients, ICP can be controlled with weight loss and with drugs that reduce cerebrospinal fluid (CSF) production. ${ }^{6-8}$ In some patients, CSF divergence surgery or optic nerve sheath fenestration is necessary to prevent permanent visual loss. ${ }^{9}$ In 2018 , the first consensus guidelines for investigation and management of IIH were published and are summarised below. ${ }^{10}$

Authors: ${ }^{\text {A }}$ consultant neurologist with a specialist interest in headache, Gloucestershire Royal Hospital, Gloucester, UK; ${ }^{B}$ consultant ophthalmologist, University Hospitals Birmingham NHS Foundation Trust, Birmingham, UK; ' professor of neurology, University Hospitals Birmingham NHS Foundation Trust, Birmingham, UK and University of Birmingham, Edgbaston, UK

\section{Diagnosis and investigation}

History

Most patients with IIH present either with new onset or worsening headache, or are found by their optician incidentally to have papilloedema. ${ }^{11}$ The headache in IIH frequently resembles migraine and often occurs daily. ${ }^{12}$ There may be 'high pressure' headache symptoms too, with headache present on waking and worsening with manoeuvres that increase ICP further by Valsalva (eg coughing). ${ }^{13,14}$ Visual disturbance or loss is also common and may include transient momentary darkening of vision on standing or bending (visual obscurations); horizontal diplopia secondary to abducens nerve palsy; reduced peripheral vision; or blurred vision with loss of acuity and colour sensitivity. ${ }^{15}$ A proportion

\section{Key points}

Identification of papilloedema is a common source of error in the diagnosis of idiopathic intracranial hypertension (IIH); it is both under- and over-diagnosed. Therefore, where doubt exists, ensure that there is a specialist doctor confirming its presence.

Computed tomography venography or magnetic resonance venography should be performed alongside neuroimaging to exclude a cerebral venous sinus thrombosis.

There is not a single cut off in lumbar puncture opening pressure that is pathological. The UK specialists that manage those with IIH defined a grey zone between 250-300 mmCSF to which they consider clinically to be disease defining.

For those with rapidly progressive visual loss (termed fulminant IIH), surgical intervention is required to save sight.

Migraine-like headache occurs in the majority of patients with IIH and often responds to preventative medication used to treat migraine.

KEYWORDS: Idiopathic intracranial hypertension, headache obesity, papilloedema, raised intracranial pressure

DOI: 10.7861/clinmed.2020-0232 


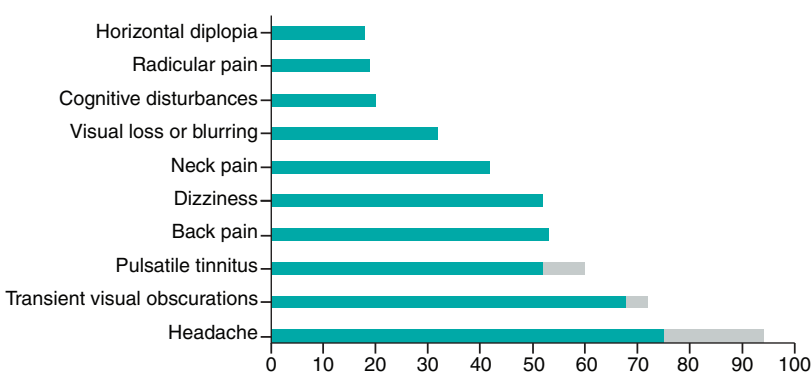

Fig 1. Most common symptoms in idiopathic intracranial hypertension.

also complain of pulsatile tinnitus (whooshing ear noises in time with their heart beat), which is worse on lying down. Other symptoms include dizziness, mild cognitive disturbance and upper cervical or radicular pain (Fig 1). ${ }^{10,16}$ Often there is a history of increased weight gain. Rarely ICP can be raised secondary to other medical conditions, which should also be considered, especially if the patient is of normal weight. ${ }^{10}$ The commonest differential diagnoses include iron deficiency anaemia; cerebral venous sinus thrombosis; medications such as fluoroquinolones and tetracyclines; and vitamin A analogues. ${ }^{10,17}$

\section{Examination}

If papilloedema is suspected, then we recommend emergency referral to secondary care. A formal visual assessment should be conducted including visual acuity, colour vision, pupils and visual field assessment. ${ }^{15}$ An extraocular movement examination should be performed to exclude oculomotor cranial nerve palsy/ palsies. Patients with longstanding IIH may have optic atrophy. Ocular imaging documents optic nerve head changes (Fig 2). Optical coherence tomography (OCT) allows non-invasive 3D representation of retinal structures. It may aid the distinction of papilloedema from pseudopapilloedema, but it is most useful for longitudinal, quantified monitoring of optic nerve head swelling and is therefore ideal for patient follow-up (Figs 2 and 3$)^{18}$ The neurological examination should otherwise be normal. Malignant hypertension (diastolic blood pressure $\geq 120 \mathrm{mmHg}$ or systolic blood pressure $\geq 180 \mathrm{mmHg}$ ) should be excluded. ${ }^{10}$

\section{Investigations}

If papilloedema is confirmed, then it is essential to exclude intracranial causes of raised ICP. Appropriate and timely brain imaging is therefore essential. All patients with confirmed papilloedema should have urgent magnetic resonance imaging (MRI) or computed tomography (CT) of the head with MR or CT venography within 24 hours. ${ }^{10}$ Certain neuroimaging characteristics of raised ICP are frequently observed in IIH (Fig 4) and, when present, support diagnosis but in isolation are not diagnostic. ${ }^{19}$ Once brain imaging has been confirmed as normal, the next step is to assess CSF opening pressure by lumbar puncture (LP) manometry. CSF constituents should be normal. Opening pressure is measured with the patient in the lateral decubitus position and, if possible, with their legs extended. Breath holding, hyperventilation and Valsalva should be avoided as these may transiently change ICP. LP is difficult in obese patients and ultrasound can help to facilitate the procedure. ${ }^{20,21}$ If LP is unsuccessful, then it could be done under $\mathrm{X}$-ray guidance. A diagnosis of IIH requires CSF opening pressure greater than 250 mmCSF (Box 1). ${ }^{22}$ If the CSF opening pressure is below $250 \mathrm{mmCSF}$, but there is strong clinical suspicion of IIH, then repeating LP examination may be informative.

Currently there is no evidence how much CSF should be removed or what the closing pressure should be. Most patients experience some improvement in their headache following $L P$, but the benefit is small and often short lived. Post-LP headache exacerbation is common and in some prolonged and severe. ${ }^{23}$ The IIH guidelines do not recommend therapeutic $\mathrm{LP}$ in $\mathrm{IIH}^{10}$

\section{Treatment}

Management of IIH should focus on treatment of the underlying disease, protection of vision and reduction of headache morbidity. $^{10,24}$

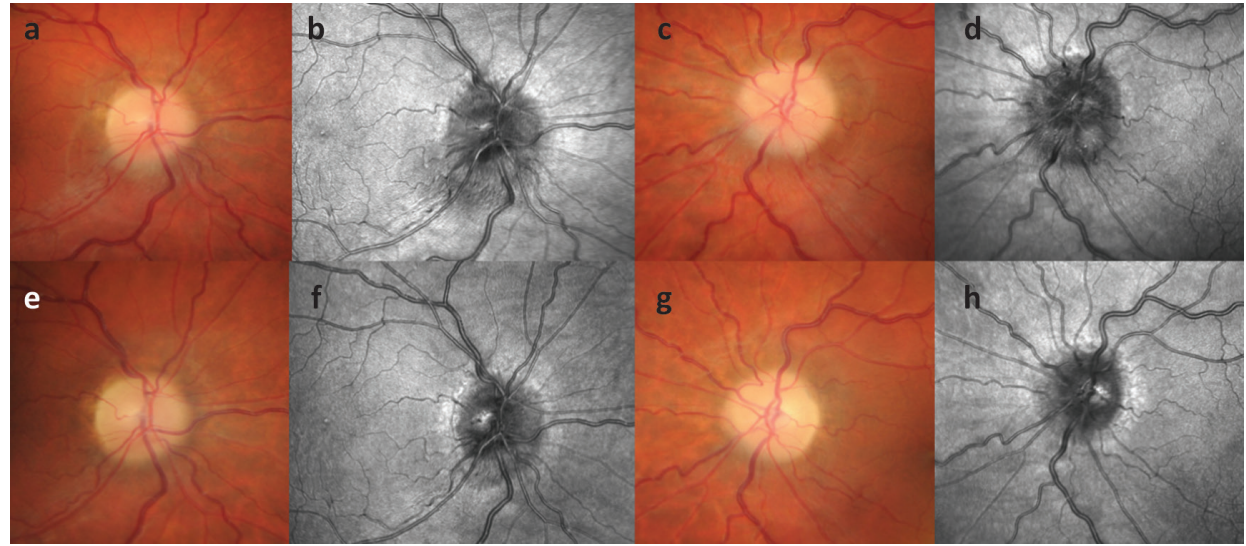

Fig 2. Progressive optical coherence tomography of retinal structures. This patient presented with papilloedema (a-d) was managed locally and re-referred back to our services following weight loss at 14 months with resolving papilloedema (e-h). The images show the difference between colour fundus images (a, c, e, g) and infrared images ( $b, \mathrm{~d}, \mathrm{f}, \mathrm{h}$ ) taken using Heidelberg Spectralis optical coherence tomography. $\mathrm{a}$ ) The right optic nerve head showing swelling at presentation. b) Greater fidelity image of the swelling of right optic nerve head. c) The left optic nerve head showing swelling at the presentation. d) Comparative image showing left optic nerve head swelling. e) The right optic nerve head showing resolving of the swelling compared with (a). f) Image showing the reduction in swelling compared with (b). g) The left optic nerve head showing resolving of swelling compared with (c). h) Image showing resolving of swelling compared with (d). 


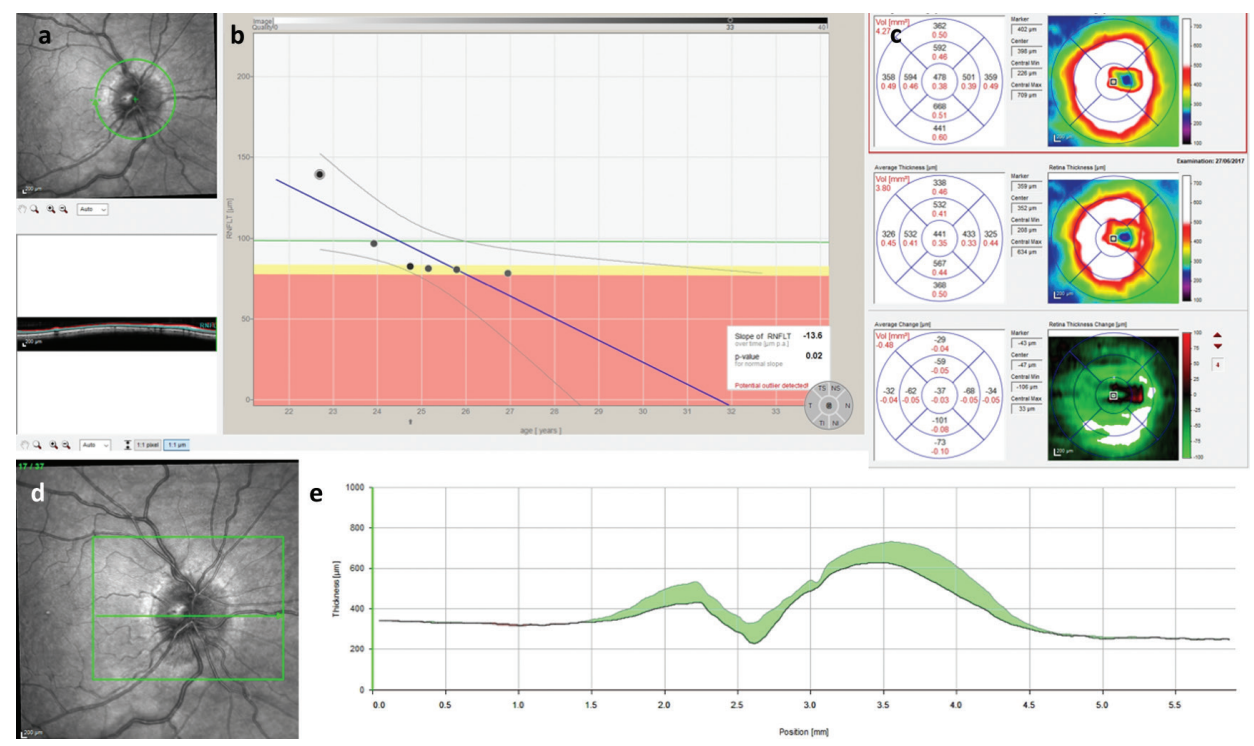

Fig 3. Scans used to longitudinally monitor papilloedema from the same patient as in Fig 2. a) Infrared (IR) imaging showing the peripapillary retinal nerve fibre layer (RNFL), the green circle shows where data are taken from. b) Resolution over time in the height of the RNFL. c) The volume of the optic nerve head showing changes from the reference scan (top) to the present scan (middle) and the average change in microns (bottom). d) IR imaging and the volume cube scan taken over the optic nerve head. e) The individual line scan for one of the middle slices through the optic nerve head, the fine grey line shows the original extent of the swelling of the right optic nerve, the black shows the present scan and the green band shows the amount of reduction in the swelling.

\section{Modifying the underlying disease}

Weight loss is disease modifying in IIH and can induce disease remission. ${ }^{8}$ Weight loss of $15 \%$ in IIH significantly reduces papilloedema and headache. ${ }^{6}$ For many patients, weight loss is challenging to achieve and maintain. Patients in the UK NHS with body mass index $\geq 35 \mathrm{~kg} / \mathrm{m}^{2}$ are currently eligible to be referred for weight management, with the aim of reducing weight by $10-15 \%$. Trials are underway to determine whether patients with IIH benefit from weight loss surgery. ${ }^{25}$

\section{Medical therapy}

Acetazolamide inhibits carbonic anhydrase and therefore reduces CSF production and is the most commonly prescribed drug. Despite widespread use of acetazolamide, a recent Cochrane review could not recommend or reject its use based on current clinical trial data. ${ }^{7,26,27}$ When prescribing, a typical acetazolamide dosage is $250 \mathrm{mg}$ twice a day, titrated according to benefits and tolerability to a maximum of $4 \mathrm{~g}$ per day. ${ }^{10}$ Patients should be warned of possible side effects, including diarrhoea, vomiting, low mood, paraesthesia, renal stones and teratogenicity. Acetazolamide is often poorly tolerated and patients are frequently switched to topiramate, which also inhibits carbonic anhydrase and has the additional benefits of appetite suppression and migraine prevention, although there is no randomised evidence of efficacy in IIH. ${ }^{8,26}$ It is typically started at a dose of 25 mg once daily and increased by $25 \mathrm{mg}$ every couple of weeks until achieving a maintenance dose of $50 \mathrm{mg}$ twice a day. ${ }^{10}$ In addition to reducing the effectiveness of the contraceptive pill, topiramate is teratogenic ( $3 \%$ risk of major malformations) and should be avoided in pregnancy. Other common adverse events include low mood and cognitive slowing. Rarely it can cause acute glaucoma and renal stones. Novel therapeutic options such as GLP-1 receptor agonists are being trialled in IIH as they reduce CSF secretion and can have anti-obesity effects. ${ }^{28,29}$

There is increasing opinion that headache in IIH should be managed as chronic migraine. ${ }^{10}$ Providing there are no contraindications, patients should therefore be trialled on migraine preventatives, including topiramate or candesartan. More commonly prescribed migraine preventatives may exacerbate low mood or induce weight gain and therefore these side effects limit their use in IIH. Medication should be started at the lowest possible dose and slowly titrated according to benefits and tolerability. If there has not been a significant reduction in headache after 3 months at the maximum tolerated dose, then alternatives should be trialled. It remains unclear whether botulinum toxin or the newer anti-calcitonin gene-related peptide monoclonal antibodies have a role. Up to a third of patients with IIH develop medication-overuse headache. ${ }^{30,31}$ Patients should therefore be warned not to take painkillers, especially opiates, on more than 2 or 3 days in a given week.

\section{Surgical treatment}

Some patients with IIH develop permanent visual damage or even go blind. ${ }^{10,15}$ Male patients, and those with severe papilloedema and reduced visual acuity at presentation, are more likely to fail medical therapy. ${ }^{32}$ In patients who develop rapidly progressive visual loss (fulminant IIH), surgical intervention is required in order to save sight. ${ }^{2}$ Ventriculoperitoneal shunts are favoured to divert CSF; however shunt complications are common and therefore should only be offered if vision is at risk and not in patients with stable IIH or to treat headache. ${ }^{9}$ Optic nerve sheath fenestration is an alternative, when there is the appropriate surgical expertise. Corticosteroids are not advised in patients with rapidly progressive 


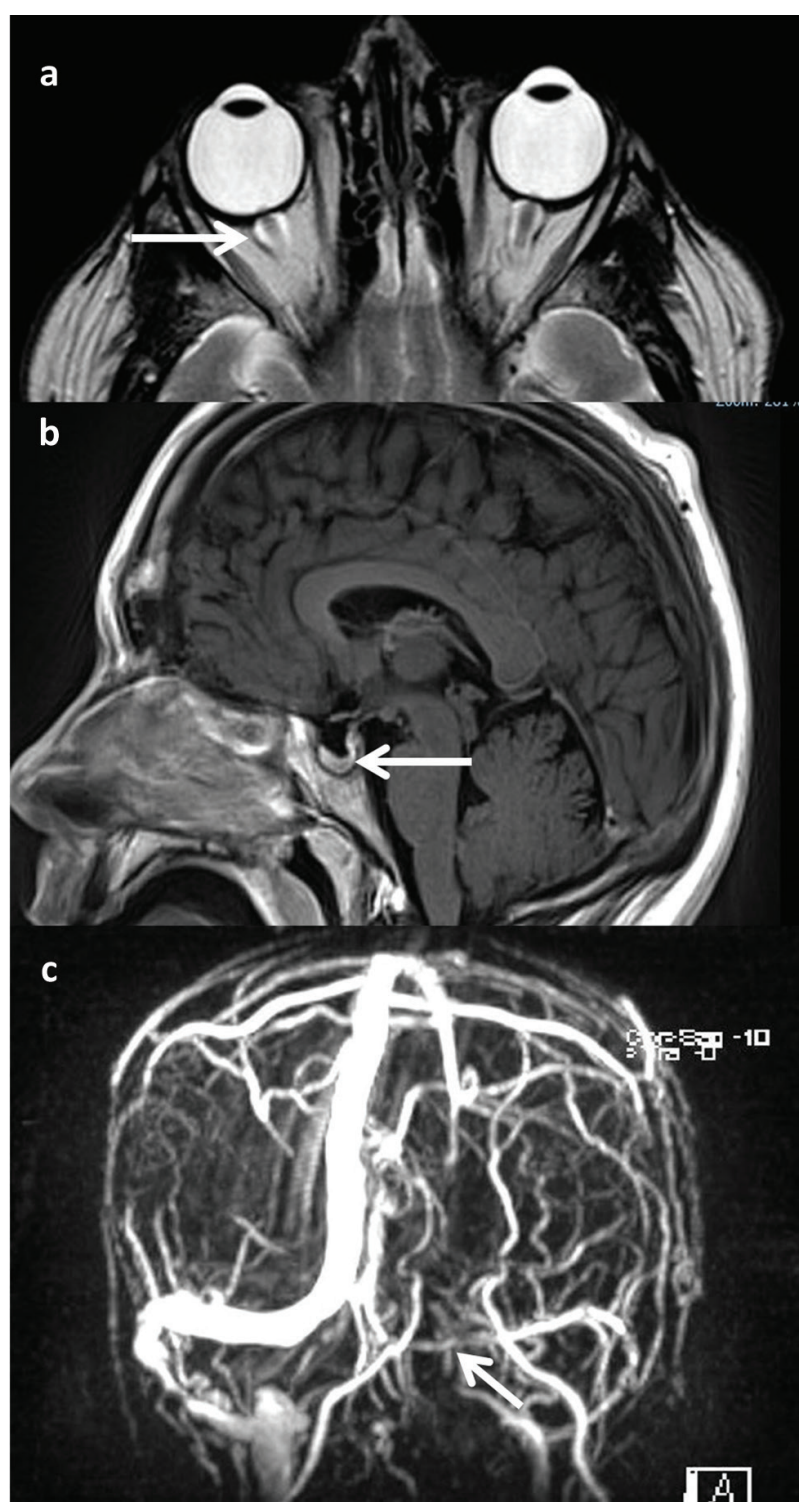

Fig 4. Typical radiological features of stigmata of raised intracranial pressure. a) Axial T2-weighted brain magnetic resonance imaging (MRI) demonstrating widening of the perioptic spaces (arrow), which are normally barely visible in healthy individuals. b) Flattening of the anterior pituitary gland by increased cerebrospinal fluid pressure resulting in a partially empty sella (arrow), which is best appreciated on sagittal T1-weighted brain MRI. c) Left extrinsic compression of the transverse sinus demonstrated here with MRI venography (arrow).

visual loss and may cause weight gain. ${ }^{10}$ It remains uncertain whether endovascular stenting helps in the longer term. ${ }^{33}$

\section{Follow-up}

All patients with confirmed IIH should be referred to both the neurology and ophthalmology teams. Follow-up depends on baseline visual assessment and stability of vision. ${ }^{10}$ For example; patients with severe papilloedema and stable visual field assessment should be followed weekly, whereas those with mild papilloedema and stable vision should attend every 6 months. ${ }^{10}$
Box 1. For a diagnosis of idiopathic intracranial hypertension to be made, all five criteria must be met to fulfil the modified diagnostic criteria ${ }^{22}$

$>$ Papilloedema present.

> Normal neurologic examination except for cranial nerve abnormalities (typically VI nerve/s).

> Normal neuroimaging: no evidence of hydrocephalus, mass or structural lesion, and no abnormal meningeal enhancement on magnetic resonance imaging. Typical radiological features of stigmata of raised intracranial pressure are shown in Fig 4.

> Normal cerebrospinal fluid composition.

> Raised lumbar puncture opening pressure ( $\geq 250 \mathrm{mmCSF}$ in adults and $\geq 280 \mathrm{mmCSF}$ in children ( $250 \mathrm{mmCSF}$ if the child is not sedated and not obese)) in a properly performed lumbar puncture.

Once in remission and with papilloedema having disappeared, we recommend ongoing follow-up as IIH can recur, particularly in relation to weight gain.

\section{Prognosis and complications}

Low mood and anxiety, obstructive sleep apnoea and polycystic ovarian syndrome frequently co-exist in IIH and should be appropriately managed by a specialist. ${ }^{10} \mathrm{IIH}$ is rare and therefore patients may benefit from speaking to other patients with the condition and reading patient information literature (for example at www.IIH.org.uk).

Patients with IIH have double the normal risk of cardiovascular disease and therefore modifiable risk factors (eg cessation of smoking) should be addressed early. ${ }^{5}$

Patients with IIH occasionally deteriorate or relapse, especially following rapid weight gain. Therefore, patients need help to find long-term solutions for maintenance of weight loss. ${ }^{25}$ Special advice during pregnancy is important, for example acetazolamide and topiramate should be stopped. Those who develop IIH in pregnancy need enhanced follow-up and advice on appropriate and safe levels of weight gain during each trimester, as recommended by the World Health Organization. ${ }^{34}$ Often the disease settles and there is no requirement for an assisted delivery once papilloedema is mild and a prolonged second stage of labour is not anticipated. ${ }^{10}$

\section{Conclusions}

IIH is a rare and poorly understood condition, which appears to be closely associated with obesity. Correct diagnosis of papilloedema and early exclusion of other intracranial causes of raised ICP are essential. LP pressure measurement is one of the five requirements to confirm diagnosis of IIH, and therefore should not be considered in isolation to the other clinical and investigation criteria (Box 1). Long-term treatment of IIH is best achieved with weight loss, although in the shorter term, drugs that reduce CSF production may be useful and surgical intervention is sometimes required to save vision in patients who are rapidly deteriorating. The headache phenotype in IIH is often migraine and patients may benefit from migraine abortive and preventative medications. ${ }^{14}$ Management of IIH is best achieved by adopting a multidisciplinary team approach with neurologists and ophthalmologists with regular follow-up dependent on visual status and headache. ${ }^{10}$ 


\section{Conflicts of interest}

Benjamin Wakerley has received an educational grant from Novartis in the past. He has received consulting fees from Invex Therapeutics in relation to developing therapeutics for intracranial pressure. Susan Mollan sits on an advisory board for therapeutics. Alexandra Sinclair has received honorary from Allergan and Novartis for talks on the topic of migraine and taken part in an advisor board on the topic of headache for Novartis. She has received consulting fees and share options from Invex Therapeutics in relation to developing therapeutics for intracranial pressure.

\section{References}

1 Markey KA, Mollan SP, Jensen RH, Sinclair AJ. Understanding idiopathic intracranial hypertension: mechanisms, management, and future directions. Lancet Neurol 2016;15:78-91.

2 Mollan SP, Aguiar M, Evison F, Frew E, Sinclair AJ. The expanding burden of idiopathic intracranial hypertension. Eye (Lond) 2019; 33:478-85.

3 Hornby C, Mollan SP, Botfield H, O'Reilly MW, Sinclair AJ. Metabolic concepts in idiopathic intracranial hypertension and their potential for therapeutic intervention. J Neuroophthalmol 2018;38:522-30.

4 O'Reilly MW, Westgate CS, Hornby C et al. A unique androgen excess signature in idiopathic intracranial hypertension is linked to cerebrospinal fluid dynamics. JCI Insight 2019;4:e125348.

5 Adderley NJ, Subramanian A, Nirantharakumar K et al. Association between idiopathic intracranial hypertension and risk of cardiovascular diseases in women in the united kingdom. JAMA Neurol 2019;76:1088-98.

6 Sinclair AJ, Burdon MA, Nightingale PG et al. Low energy diet and intracranial pressure in women with idiopathic intracranial hypertension: prospective cohort study. BM] 2010;341:c2701.

7 NORDIC Idiopathic Intracranial Hypertension Study Group Writing Committee, Wall M, McDermott MP et al. Effect of acetazolamide on visual function in patients with idiopathic intracranial hypertension and mild visual loss: the idiopathic intracranial hypertension treatment trial. JAMA 2014;311:1641-51.

8 Scotton WJ, Botfield HF, Westgate CS et al. Topiramate is more effective than acetazolamide at lowering intracranial pressure. Cephalalgia 2019;39:209-18.

9 Bjornson A, Tapply I, Nabbanja E et al. Ventriculo-peritoneal shunting is a safe and effective treatment for idiopathic intracranial hypertension. Br J Neurosurg 2019;33:62-70.

10 Mollan SP, Davies B, Silver NC et al. Idiopathic intracranial hypertension: consensus guidelines on management. J Neurol Neurosurg Psychiatry 2018;89:1088-100.

11 Blanch RJ, Vasseneix C, Liczkowski A et al. Differing Presenting Features of Idiopathic Intracranial Hypertension in the UK and US. Eye (Lond) 2019:33:1014-9.

12 Friedman DI, Quiros PA, Subramanian PS et al. Headache in idiopathic intracranial hypertension: findings from the idiopathic intracranial hypertension treatment trial. Headache 2017:57:1195-205.

13 Mollan SP, Spitzer D, Nicholl DJ. Raised intracranial pressure in those presenting with headache. BMJ 2018;363:k3252.

14 Mollan SP, Hoffmann J, Sinclair AJ. Advances in the understanding of headache in idiopathic intracranial hypertension. Curr Opin Neurol 2019;32:92-8.

15 Mollan SP, Markey KA, Benzimra JD et al. A practical approach to, diagnosis, assessment and management of idiopathic intracranial hypertension. Pract Neurol 2014;14:380-90.

16 Wall M, Kupersmith MJ, Kieburtz KD et al. The idiopathic intracranial hypertension treatment trial: clinical profile at baseline. JAMA Neurol 2014;71:693-701.

17 Mollan SP, Ball AK, Sinclair AJ et al. Idiopathic intracranial hypertension associated with iron deficiency Anaemia: a lesson for management. Eur Neurol 2009;62:105-8.
18 Mitchell JL, Mollan SP, Vijay V, Sinclair AJ. Novel advances in monitoring and therapeutic approaches in idiopathic intracranial hypertension. Curr Opin Neurol 2019;32:422-31.

19 Wong H, Sanghera K, Neufeld A, Maxner C, Shankar JJS. Clinicoradiological correlation of magnetic resonance imaging findings in patients with idiopathic intracranial hypertension. Neuroradiology 2020;62:49-53.

20 Williams S, Khalil M, Weerasinghe A, Sharma A, Davey R. How to do it: bedside ultrasound to assist lumbar puncture. Pract Neurol 2017:17:47-50.

21 Scotton W], Mollan SP, Walters T et al. Characterising the patient experience of diagnostic lumbar puncture in idiopathic intracranial hypertension: a cross-sectional online survey. BMJ Open 2018:8:e020445.

22 Friedman DI, Liu GT, Digre KB. Revised diagnostic criteria for the pseudotumor cerebri syndrome in adults and children. Neurology 2013:81:1159-65.

23 Yiangou A, Mitchell J, Markey KA et al. Therapeutic lumbar puncture for headache in idiopathic intracranial hypertension: Minimal gain, is it worth the pain? Cephalalgia 2019;39:245-53.

24 Mollan SP, Hornby C, Mitchell J, Sinclair AJ. Evaluation and management of adult idiopathic intracranial hypertension. Pract Neurol 2018;18:485-8.

25 Ottridge R, Mollan SP, Botfield $\mathrm{H}$ et al. Randomised controlled trial of bariatric surgery versus a community weight loss programme for the sustained treatment of idiopathic intracranial hypertension: the Idiopathic Intracranial Hypertension Weight Trial (IIH:WT) protocol. BMJ Open 2017;7:e017426.

26 Ball AK, Howman A, Wheatley $\mathrm{K}$ et al. A randomised controlled trial of treatment for idiopathic intracranial hypertension. J Neurol 2011;258:874-81.

27 Piper RJ, Kalyvas AV, Young AM et al. Interventions for idiopathic intracranial hypertension. Cochrane Database Syst Rev 2015: CD003434.

28 Markey K, Mitchell J, Botfield $\mathrm{H}$ et al. 11 $\beta$-Hydroxysteroid dehydrogenase type 1 inhibition in idiopathic intracranial hypertension: a double-blind randomized controlled trial. Brain Communications 2020;2:fcz050.

29 Botfield HF, Uldall MS, Westgate CS] et al. A glucagon-like peptide-1 receptor agonist reduces intracranial pressure in a rat model of hydrocephalus. Sci Transl Med 2017;9:eaan0972.

30 Wakerley BR. Medication-overuse headache. Pract Neurol 2019; 19:399-403.

31 Lai JT, Dereix JD, Ganepola RP et al. Should we educate about the risks of medication overuse headache? J Headache Pain 2014;15:10

32 Wall M, Falardeau J, Fletcher WA et al. Risk factors for poor visual outcome in patients with idiopathic intracranial hypertension. Neurology 2015;85:799-805.

33 Gurney SP, Ramalingam S, Thomas A, Sinclair AJ, Mollan SP. Exploring the current management idiopathic intracranial hypertension, and understanding the role of dural venous sinus stenting. Eye Brain 2020;12:1-13.

34 World Health Organization. 5.2 - Recommendations for preventing excess weight gains and obesity. In: Diet, nutrition and the prevention of chronic diseases: Report of the joint WHO/FAO expert consultation: WHO Technical Report Series, No. 916 (TRS 916). WHO, 2003:61-71. www.who.int/dietphysicalactivity/publications/ trs916/en/gsfao_obesity.pdf?ua=1.

Address for correspondence: Prof Alexandra J Sinclair, Metabolic Neurology, Institute of Metabolism and Systems Research, University of Birmingham, Edgbaston, Birmingham B15 2TT, UK.

Email: a.b.sinclair@bham.ac.uk 